\title{
SEE YOUR ENDOCRINOLOGIST FOR PITUITARY TUMOUR; YOU MAY NOT NEED SURGERY
}

\author{
Ahmed Imran Siddiqi \\ Department of Internal Medicine, Shaukat Khanum Memorial Cancer Hospital and Research Centre, Lahore, \\ Pakistan
}

Received: 17 January 2017 / Accepted 15 March 2017

\begin{abstract}
This brief review emphasises the importance of proper diagnosis before management of patients with pituitary lesions. Surgery remains the treatment of choice for many pituitary adenomas but not all of them require surgery. Lactotroph adenomas secreting an excessive amount of prolactin (often termed as prolactinomas) are usually responsive to pharmacological treatment with dopamine receptor (D2) agonists and should not be treated surgically except in a selected group of patients. Surgery is no longer the first-line treatment for prolactinomas. All those patients of prolactinomas who may be considered for surgery must be discussed in a multidisciplinary team meeting before surgical intervention. In clinical practice in Pakistan, one comes across a noticeable number of patients of lactotroph adenomas referred after surgical resection performed as a primary treatment for their pituitary adenomas. Most of these patients were referred to the endocrinology department following a recurrence of prolactinomas, and further, surgical resection could not be performed. There are no local data of those prolactinomas which may have been cured after surgery. The comparison between surgical and pharmacological treatment is not possible in local population but worldwide data available in literature suggests that surgery should no longer be the first-line treatment in the majority of patients with prolactinomas. Pharmacological treatment is convenient, more effective and longer lasting compared to surgical treatment.
\end{abstract}

Key words: Cabergoline, dopamine agonists, hyperprolactinemia, lactotroph adenoma, pituitary adenoma, prolactinoma

\section{Introduction}

This brief review is aimed at reviewing basic aspects of the management of prolactinomas for health-care professionals of all specialities and grades. There seems to be a lack of awareness about prolactinomas not only amongst patients but also amongst some of the healthcare professionals in Pakistan. Most patients present late to a doctor, but even after the presentation, they are not referred to an appropriate centre immediately. Lack of availability of the appropriate laboratory facilities in most of the hospitals further emphasises the importance of timely referral to appropriate centres for investigation and management of these patients. Furthermore, many of these patients may not present to general practitioners but may present to gynaecologists (with amenorrhoea)

Correspondence: Ahmed Imran Siddiqi, Department of Internal Medicine, Shaukat Khanum Memorial Cancer Hospital and Research Centre, Lahore, Pakistan.

Email: ahmedimran@skm.org.pk and ophthalmologists (visual field defects). In essence a multidisciplinary approach is required for the best management of such patients.

Prolactin is a single chain polypeptide hormone of 198 amino acids and approximately $24,200 \mathrm{D}^{\left[{ }^{[1]}\right.}$ It is produced and secreted by the acidophilic cells of the anterior pituitary gland. Most of the anterior pituitary gland hormones secretion is regulated by stimulatory and inhibitory effects of hypothalamic hormones. Prolactin production and secretion by lactotroph cells are inhibited by dopamine produced in the hypothalamus and released in hypophyseal-hypothalamic circulation. All the stimulating factors for prolactin are not fully understood, but thyrotropin-releasing hormone produced in the hypothalamus is one of the prolactin stimulatory agents. This explains the reason for high prolactin level in some patients of primary hypothyroidism. Lactation has almost always been considered as its main function, and the hormone has been named accordingly, ${ }^{[2]}$ but this is not 
the only functions it performs in mammary glands. It has a trophic effect on the secretory epithelial tissue of the mammary glands. This trophic effect results in growth and prepares the tissue for the initiation of lactation. Prolactin not only helps in the initiation of lactation but also then maintains it as well.

Since almost all the vertebrates produce prolactin, most of the functions of prolactin have been studied in animal models. In addition to its function in reproduction and lactation, it has also been associated with a role in metabolism, behaviour, thermoregulation and osmoregulation. Up to 300 different biological actions of prolactin have been described in vertebrates. ${ }^{[3]}$ It is important to manage abnormalities of such an important hormone properly.

\section{Presentation}

Prolactinoma is one of the many causes of hyperprolactinemia. ${ }^{[4]}$ Hyperprolactinemia could be physiological (during lactation and pregnancy) or pathological which should be investigated and treated. Hyperprolactinemia can also be transient due to a number of reasons, but clinical symptoms of hyperprolactinemia develop if it remains persistently high. Most patients with prolactinomas usually present with symptoms and signs of hyperprolactinemia or compression of pituitary tumour on the surrounding intracranial structures. Hyperprolactinemia reduces gonadotropin levels and patients often present with clinical and biochemical features of hypogonadotropic hypogonadism. In a retrospective study conducted by Peng et al. found that most adult men experience growth hormone deficiency and hypogonadism with prolactinomas. ${ }^{[5]}$ Most of these patients are young; however, older patients may also present with prolactinomas. Fernandez et al. conducted a study in 81,449 inhabitants of Banbury, Oxfordshire and in the United Kingdom and determined the incidence of pituitary adenomas there to be 77.6 cases per 100,000 population, with the majority of cases $(57 \%$, or 44.4 persons per 100,000 population) being prolactinomas.

In this particular population, the incidence of prolactinomas was $75 \%$ of all pituitary adenomas occurring in individuals up to the age of 20 years and $61 \%$ of all the pituitary adenomas in individuals between the ages of 20 years and 60 years.
The incidence of prolactinomas was higher amongst women compared to men as prolactinomas made $76 \%$ of all the pituitary adenomas in women while in men $57 \%$ were non-functioning adenomas. ${ }^{[6]}$ Carter et al. found that $60 \%$ of men present with macroprolactinomas and $90 \%$ of women present with microprolactinomas. ${ }^{[7]}$ Women present early with menstrual irregularities. It can present with oligomenorrohea, amenorrohea or irregular menstrual bleeding. ${ }^{[8]}$ Patients can sometimes present with secondary amenorrohea or primary amenorrohea. Hyperprolactinemia is often detected during work up in infertility clinics. ${ }^{[8]}$ Women may also experience vaginal dryness, dyspareunia and bone mineral density (BMD) loss secondary to hypoestrogenemia secondary to hypogonadotropic hypogonadism. ${ }^{[4]}$ Men with prolactinomas usually present late. They present with either compression effects on surrounding structures or deficiency of other pituitary hormones. Since hypogonadotropic hypogonadism develops gradually over time, most patients may not notice this until the symptoms are fairly advanced. Many such patients present for the $1^{\text {st }}$ time with visual field defects due to compression of tumour on optic chiasm.

Large tumours may affect other surrounding structures in the vicinity including III, IV and VI cranial nerves. Many patients present with headache with large tumours stretching onto to pain sensitive surrounding structures. Men could present with erectile dysfunction, low libido, loss of BMD and infertility. ${ }^{[7]}$ Galactorrohea remains the other most commonly experienced symptom amongst such patients. Approximately $30-60 \%$ patients experience this symptom with hyperprolactinemia. Gynaecomastia is not a typical sign in such patients although it has been reported to be associated with some patients of hyperprolactinemia. Pre-pubertal hyperprolactinemia can result in female body habitus in male patients due to hypogonadotrophic hypogonadism. Incidental detection of pituitary adenomas has increased in recent years with an increased number of computed tomography and magnetic resonance imaging (MRI) scans. Prolactinomas constitute a reasonable proportion of these incidentally detected pituitary adenomas. Larger adenomas can cause a deficiency of other pituitary hormones in addition to gonadotropins. Cortisol and thyroxin must be replaced once their deficiency is confirmed. Cortisol has to be replaced before thyroxin replacement to avoid an 
adrenal crisis. Prolactinomas can also present along with other endocrine neoplasias termed as multiple endocrine neoplasia (MEN). Prolactinomas associated with parathyroid and pancreatic tumours are termed as MEN Type 1 . Such patients usually present early in their lives with symptoms of one or more hormones excess; parathyroid in $90 \%$, prolactin in $66 \%$ and gut hormones excess in $50 \%$ patients. In younger patients, it is advisable to assess other hormones (pituitary, gut hormones and parathyroid) once an adenoma is detected in pancreas, pituitary or parathyroid glands.

\section{Laboratory Assessment}

Most laboratories use immunoassay as the first line investigation for prolactin level assessment. Prolactin immunoassay is more like antigen-antibody reaction. Prolactin molecule in sample acts as antigen while antiprolactin antibodies in assay attach to prolactin. One prolactin molecule is sandwiched between two antibodies. This suggests that laboratory assay should have more antibodies than antigen to reliably detect prolactin concentration in a given blood sample. If the antigen concentration increases significantly, the laboratory assay antibodies may not be able to sandwich prolactin molecules properly. ${ }^{[9,10]}$ In this situation, a significant proportion of prolactin is attached to only one antibody and is not detected. This leaves fewer antibodies in the assay to be attached on two antigen sites (sandwich prolactin molecule) of prolactin and be detected. This will result in a falsely low detectable prolactin level in a given blood sample. This is called 'hook effect' when very high levels of prolactin in a blood sample result in a falsely low detectable prolactin and hook the result in the wrong direction. One could overcome this phenomenon by either reducing the concentration of antigen or increasing concentration of antibody. One way of reducing antigen concentration would be to dilute the blood sample. Normally with dilution, you would expect the detectable prolactin levels to drop; however, with hook effect sample the prolactin levels will be higher in diluted sample. The dilution of the blood sample is continued to be increased in a stepwise manner as long as the prolactin level continues to rise with increasing sample dilution. Once the prolactin level starts to drop with increasing dilution further dilutions are not necessary. The corrected level of prolactin is then documented by multiplying it with the number of times the blood sample was diluted. The immunoradiometric PRL assay must be performed with serum dilution to overcome the high-dose PRL hook effect in all new patients with pituitary macroadenomas who may have a prolactinoma. ${ }^{[11]}$ Dilution of sample is especially recommended when there is a discrepancy between pituitary tumour size and prolactin levels. The hook effect was first described in $1992^{[12]}$ and Comtois et al. ${ }^{[13]}$ reported the first patient in whom the hook effect lead to misdiagnosis of a large macroprolactinoma. Subsequently, a good number of cases was reported in literature with hook effect. It is important to recognise this phenomenon for correct diagnosis and treatment of pituitary tumours.

Most laboratories usually assess total prolactin as the initial investigation in all patients. Once the immunoassay detects high prolactin level further workup is required to assess if the prolactin is biologically active. Assessment of bio-active (monomeric) prolactin is the laboratory investigation of choice in patients with clinical features of hyperprolactinemia and/or pituitary tumour. Most of human circulating prolactin exists in a monomeric form of $23 \mathrm{kDa}(85-90 \%)$. A proportion of prolactin circulates as high molecular mass isoforms including big-PRL, a dimer of $50 \mathrm{kDa}$ and big-big-PRL or macroprolactin, a variant of $150-170 \mathrm{kDa} \cdot{ }^{[14]}$ It is believed that macroprolactin is formed when prolactin binds to a protein $\mathrm{IgG}$ (immunoglobulin $\mathrm{G}$ ) in circulation. This IgG-prolactin complex becomes bigger in size and becomes biologically inactive although stays in circulation for longer than monomeric prolactin. Posttranslational modification of pituitary prolactin with varying glycosylation and phosphorylation degrees has also been suggested as a possible cause of macroprolactin. ${ }^{[15,16]}$ Macroprolactin usually does not cause clinical symptoms and does not require any treatment. There are only a few case reports suggesting clinical symptoms with high levels of macroprolactin, but an overwhelming majority of patients with macroprolactin are asymptomatic and do not require treatment. The exact mechanism of inactivity of macroprolactin is not known, but the most widely believed theory is about the size of IgG-prolactin complex. IgGprolactin complex becomes too big to cross the vessel wall and not released into tissues to produce any biological effects. Prolactin part of prolactin-IgG complex maintains its immunoreactivity and is detected during laboratory 
assays. The gold standard test to determine the presence of macroprolactinemia is gel filtration chromatography, but more available and less expensive alternatives have been described, like precipitation with polyethylene glycol. ${ }^{[17]}$

In addition to prolactin other hormones of anterior pituitary gland should be assessed regardless of prolactin level. This should include lutenizing hormone, folliclestimulating hormone, testosterone (for men), estradiol (for women), cortisol with or without adrenocorticotrophic hormone, insulin-like growth factor 1 (IGF-1), thyroidstimulating hormone and free thyroxin 4. Certain pituitary adenomas can co-secrete hormone, especially growth hormone and prolactin. All patients with high prolactin level should have their IGF-1 level assessed to look for this possibility of co-secretion.

\section{Treatment}

Once the diagnosis is confirmed treatment is planned according to biochemical and radiological findings. Prolactinomas are divided into two main sub-types based on their size: Microprolactinoma (tumour $<1 \mathrm{~cm}$ in size on radiologic imaging) and macroprolactinoma (tumour over $1 \mathrm{~cm}$ in size). This size based divide helps in planning treatment of these adenomas. The exact aetiology of micro- and macro-prolactinomas is not known; however, microprolactinomas generally do not grow in size to become macroprolactinomas in $95 \%$ of cases. Hence, the treatment is tailored according to tumour size and behaviour. One of the following approaches can be used to treat prolactinomas:

1. Monitoring without active intervention

2. Pharmacological and surgical intervention

3. Others, for example, radiotherapy.

\section{Monitoring without Intervention}

Asymptomatic microprolactinomas with stable prolactin levels and no abnormality of any of the other pituitary hormones do not always require intervention and these prolactinomas can sometimes be monitored with prolactin levels and size on pituitary MRI scans. Pharmacological treatment will be indicated if prolactin level continues to raise, other pituitary hormones are affected and/or prolactinoma increases in size. Furthermore, some infertility centres recommend normalisation of prolactin levels in women before in vitro fertilisation treatment cycle to maximize chances of ovulation. In the absence of any of these conditions radiological and biochemical monitoring is advised on 3-6 monthly intervals. Many patients of polycystic ovarian syndrome have high prolactin levels with no identifiable adenoma in the pituitary gland. The exact mechanism of high prolactin in these patients in not known; however, these patients should be monitored for symptoms as above and treatment should be offered using the same criteria as those who have got prolactinomas. Since there is no detectable pituitary adenoma on MRI scan, most centres would not recommend repeating scans if two scans at least 6 months apart do not show a change in the pituitary gland. After initial two scans, another scan should be considered if there is a considerable increase in prolactin level or there are clinical signs of pituitary tumour - visual field loss.

In some cases of hyperprolactinemia no cause can be found. In the absence of any other cause of hyperprolactinemia, it is believed that the pituitary adenoma may be too small to be identified as a separate entity in pituitary gland on MRI scans. Monitoring strategy should remain the same in these patients with initial two scans at least 6 months apart and subsequent scans if required. Many of the medicines used in the treatment of psychiatric conditions can cause hyperprolactinemia. ${ }^{[18]}$ Most of these medicines are dopamine antagonists. Patients need to take these medicines to help with their psychiatric condition. The prolactin released in these conditions causes true hyperprolactinemia and can cause clinical symptoms of hyperprolactinemia. I would strongly recommend for every patient to have their prolactin level assessed before the commencement of these medications. Psychiatrists should get a baseline prolactin level on all these patients and refer to endocrinologists for assessment of those patients who have high monomeric prolactin level (unpublished; Hanson, P). Most patients will not need diagnostic workup for hyperprolactinemia if the prolactin level rises after the commencement of dopamine antagonists. A sudden significant rise in prolactin level or other signs of pituitary adenoma in such patients may require investigations for hyperprolactinemia. It is usually not possible to discontinue treatment with psychiatric medications to assess the effect of these medications on prolactin level if baseline prolactin level is not recorded before the commencement of these medications. One 
may consider switching to one of the few medications which do not increase prolactin levels; however, this is not always possible and may be risky in patients who are symptomatically controlled with a specific medication. It is generally recommended not to treat such patients with dopamine receptor agonists to control prolactin levels, but it has been done successfully in some patients to control symptoms of hyperprolactinemia with good response. ${ }^{[19]}$

Hence, many patients with pituitary microprolactinoma will not require any active treatment and monitoring alone may be sufficient. Women who wish to get pregnant during this monitoring can go ahead with pregnancy. Prolactin level rises during pregnancy and cannot be monitored during pregnancy as there are no agreed pregnancy specific values available. Such patients should have visual field assessments once every trimester during pregnancy. Breastfeeding is not contraindicated in such patients. Active intervention is indicated if there is deterioration in visual fields during pregnancy. Such patients will require individualised care following discussion with endocrinologists, neurosurgeons and obstetricians. However, the possibility of such a scenario is very low.

\section{Pharmacological Treatment}

Pharmacological treatment is indicated in symptomatic microprolactinomas, ${ }^{[20]}$ for patients with infertility associated with hyperprolactinemia and almost all macroprolactinomas. Dopamine receptor agonists are firstline agents. They are very effective not only in controlling growth of tumour but also to reduce tumour size soon after their commencement. The dose is usually gradually increased but treatment with higher doses for immediate response has been reported in literature. Bromocriptine is being used for a long time and is relatively easily available in Pakistan. It is usually started in a dose of $2.5 \mathrm{mg} 3$ times a day and can be increased at weekly intervals or according to patient response to $10-30 \mathrm{mg}$ in a day in divided doses. Multiple daily dosing regimen and gastrointestinal side effects limit its use in some patients. This is the preferred dopamine receptor agonist to be used in pregnant women or in women planning to get pregnant. Cabergoline is the other dopamine receptor agonist. It is better tolerated of the two, lower treatment failure rate, better dosing regimen and better patient experience. It is usually started at a dose of $250 \mathrm{mcg}$ (half a tablet of $500 \mathrm{mcg}$ ) once or twice a week. So far, this is not widely available in Pakistan. If possible, this should be the first-line dopamine receptor agonist used in the treatment of prolactinomas. Higher doses of dopamine receptor agonists have been associated with cardiac adverse effects in animal studies. Monitoring of cardiac functions in patients taking low doses of dopamine receptor agonists remains a debatable subject. It is suggested to get a baseline echocardiogram done on patients before the commencement of therapy and subsequently arrange more scans depending on clinical condition, dose of cabergoline or at least, once a year. Dopamine receptor agonists are used in much smaller doses in the treatment of prolactinoma and none of the studies have so far conclusively proven adverse cardiac effect in patients treated for prolactinomas with dopamine receptor agonists. Quinagolide is a non-ergot dopamine receptor (D2) agonist which can be used as a second-line agent if bromocriptine or cabergoline cannot be used. The availability of this agent remains an issue in Pakistan. The response is assessed by reduction in prolactin levels and is confirmed by repeat MRI pituitary scan in 3-6 months. It is very unlikely that there will be a reduction in tumour size if the prolactin level does not reduce with medications. In some patients, prolactin level may not drop to normal range. This is especially found with very high baseline prolactin levels. Most of these patients will become symptoms free with reduction in tumour size despite marginally high prolactin levels. These patients are not considered as treatment failure; however, if the prolactin level does not drop at all or it starts to persistently rise again following initial drop, such patients are classed as treatment failure. Cabergoline has the least of the treatment failure rate. It ranges from $10 \%$ to $13 \%$ in different studies.

\section{Surgical Treatment}

Surgery is neither the first-line treatment nor the treatment of choice for almost all prolactinomas. The success rate for microprolactinomas is $70-75 \%$, but there always remains a possibility of affecting other pituitary hormones during surgery in addition to other possible complications potentially associated with a major neurosurgical procedure. In Pakistan, a significant proportion of patients with macroprolactinomas undergo surgery as the primary treatment. The cure rate falls to only $30-35 \%$ in cases of macroprolactinomas. Assessment of prolactin level for all 
pituitary lesions remains key to diagnosis of prolactinomas and all such cases must be referred to appropriate centres with endocrinology and neurosurgical expertise.

Health-care professionals in Pakistan need to be mindful of the fact that post-surgical deficiency of other pituitary hormones on lifelong basis significantly affects lives of these patients. Neurosurgeons need to give it serious thought before attempting any kind of pituitary surgery. We may be able to cure one condition but could potentially leave them with a lifelong complication of hypopituitarism. Each hormone secreted by pituitary gland is essential for normal functioning of an individual. Cortisol deficiency is not only associated with significant morbidity but also carries mortality if not managed properly. Availability of hydrocortisone also remains an issue especially outside big cities of the country. Same is true for thyroxin in cases of secondary hypothyroidism. Not only that growth hormone cost is out of reach for most patients in Pakistan the laboratory assessment of the hormone but is also only available in a limited number of laboratories in the country. Since the majority of patients with prolactinomas are young, surgery is indicated in a selected group of patients with:

1. Prolactinoma is not responsive to pharmacological treatment or those who show the progression of disease following initial response to pharmacological treatment.

2. Patient cannot or would not take medications. ${ }^{[21,22]}$

Prolactin level may also be raised in non-functioning pituitary tumours compressing the pituitary stalk. Hypothalamus is believed to produce inhibitory effects on prolactin producing cells in pituitary gland through pituitary stalk. Suprasellar tumours or tumours with suprasellar extension compress the stalk and can block this inhibitory effect, often termed as 'stalk effect.' Treatment of primary tumour will help control prolactin levels in these patients. Prolactin level in these cases is usually not very high. Some studies have used prolactin level to differentiate between different causes of hyperprolactinemia but none of those have been conclusive enough to be used reliably in clinical practice. A prolactin level $>5.25 \mathrm{IU} / \mathrm{L}(250 \mu \mathrm{g} / \mathrm{L})$ usually indicates the presence of a prolactinoma and a prolactin level $>10.5 \mathrm{IU} / \mathrm{L}(500 \mu \mathrm{g} / \mathrm{L})$ is considered very suggestive of a macroprolactinoma. In some cases of macroprolactinomas prolactin levels are even far
$>21.2 \mathrm{IU} / \mathrm{L}(1000 \mu \mathrm{g} / \mathrm{L})$. In contrast, marginally elevated prolactin levels (usually $>2.1 \mathrm{IU} / \mathrm{L}(100 \mu \mathrm{g} / \mathrm{L})$ are very suggestive of these large tumours as non-secreting tumours. ${ }^{[23]}$

\section{Other Options}

Radiotherapy is a last resort for treatment of prolactinomas. This should only be considered a treatment option in patients who cannot undergo surgery and cannot or would not take oral medications for prolactinomas. Success rates remain very low with radiotherapy and due to this not many well-designed studies have been performed to accurately assess the success rate of this treatment option.

\section{Treatment during Pregnancy}

Many women attend endocrinology clinic with high prolactin levels and menstrual irregularities due to the effect of prolactin on their gonadotropins. This could affect ovulation and hence fertility. With medical treatment, the prolactin usually settles in normal range with normalisation of gonadotropin functions. Women with microprolactinoma are advised to discontinue their medications once pregnancy is confirmed. Medications are generally advised to be continued during pregnancy in patients with macroprolactinoma. Many clinicians would discontinue medications even in patients of macroprolactinoma if the tumour is away from optic chiasm and other vital structures. Formal visual field assessments should be carried out at least once, during each trimester. Women can breastfeed after delivery, and they are assessed after completion of breastfeeding for starting pharmacological therapy again.

\section{Conflict of Interest}

The authors declare that they have no conflict of interest.

\section{References}

1. Sabharwal P, Glaser R, Lafuse W, et al. Prolactin synthesized and secreted by human peripheral blood mononuclear cells: An autocrine growth factor for lymphoproliferation. Proc Natl Acad Sci U S A 1992;89:7713-6.

2. Riddle O, Bates RW, Dykshorn SW. The preparation, identification and assay or prolactin-a hormone of anterior pituitary. Am J Physiol 1933;105:191-216.

3. Bole-Feysot C, Goffin V, Edery M, et al. Prolactin (PRL) and its receptor: Actions, signal transduction pathways 
and phenotypes observed in PRL receptor knockout mice. Endocr Rev 1998;19:225-68.

4. Zadrozna-Sliwka B, Bolanowski M, Jawiarczyk A, et al. The role of cyclase activating (CAP) and cyclase inhibiting (CIP) parathormone fractions in the assessment of bone metabolism disturbances in women with hyperprolactinemia of various origin. Neuro Endocrinol Lett 2008;29:178-84.

5. Peng J, Qiu M, Qi S, et al. Hypopituitarism patterns among adult males with prolactinomas. Clin Neurol Neurosurg 2016;144:112-8.

6. Fernandez A, Karavitaki N, Wass JA. Prevalence of pituitary adenomas: A community-based, cross-sectional study in Banbury (Oxfordshire, UK). Clin Endocrinol (Oxf) 2010;72:377-82.

7. Carter JN, Tyson JE, Tolis G, et al. Prolactin-screening tumors and hypogonadism in 22 men. N Engl J Med 1978;299:847-52.

8. Schlechte JA. Clinical practice. Prolactinoma. N Engl J Med 2003;349:2035-41.

9. Unnikrishnan AG, Rajaratnam S, Seshadri MS, et al. The 'hook effect' on serum prolactin estimation in a patient with macroprolactinoma. Neurol India 2001;49:78-80.

10. Yener S, Comlekci A, Arda N, et al. Misdiagnosis due to the hook effect in prolactin assay. Med Princ Pract 2008; 17:429-31.

11. St-Jean E, Blain F, Comtois R. High prolactin levels may be missed by immunoradiometric assay in patients with macroprolactinomas. Clin Endocrinol (Oxf) 1996;44:305-9.

12. Haller BL, Fuller KA, Brown WS, et al. Two automated prolactin immunoassays evaluated with demonstration of a high-dose "hook effect" in one. Clin Chem 1992;38:437-8.

13. Comtois R, Robert F, Hardy J. Immunoradiometric assays may miss high prolactin levels. Ann Intern Med 1993;
119:173.

14. Silva AM, da Costa PM, Pacheco A, et al. Assessment of macroprolactinemia by polyethylene glycol precipitation method. Rev Port Endocrinol Diabetes Metab 2014;9:25-8.

15. Hattori $\mathrm{N}$. The frequency of macroprolactinemia in pregnant women and the heterogeneity of its etiologies. J Clin Endocrinol Metab 1996;81:586-90.

16. Freeman ME, Kanyicska B, Lerant A, et al. Prolactin: Structure, function, and regulation of secretion. Physiol Rev 2000;80:1523-631.

17. Fahie-Wilson MN, Soule SG. Macroprolactinaemia: Contribution to hyperprolactinaemia in a district general hospital and evaluation of a screening test based on precipitation with polyethylene glycol. Ann Clin Biochem 1997; 34(Pt 3):252-8.

18. Knegtering $\mathrm{H}$, van der Moolen AE, Castelein S, et al. What are the effects of antipsychotics on sexual dysfunctions and endocrine functioning? Psychoneuroendocrinology 2003; 28 Suppl 2:109-23.

19. Molitch ME. Medication-induced hyperprolactinemia. Mayo Clin Proc 2005;80:1050-7.

20. Serri O. Progress in the management of hyperprolactinemia. N Engl J Med 1994;331:942-4.

21. Fleseriu M, Lee M, Pineyro MM, et al. Giant invasive pituitary prolactinoma with falsely low serum prolactin: The significance of 'hook effect'. J Neurooncol 2006;79:41-3.

22. Melmed S, Casanueva FF, Hoffman AR, et al. Diagnosis and treatment of hyperprolactinemia: An endocrine society clinical practice guideline. J Clin Endocrinol Metab 2011; 96:273-88.

23. Boesten LS, Krabbe JG, de Rijke YB. The high dose hook effect in prolactinomas; Following the guidelines? Ned Tijdschr Klin Chem Labgeneesk 2015;40:230-3. 